\title{
Bulky Mediastinal Metastasis in Neglected Follicular Thyroid Carcinoma: A Case Report
}

\author{
Jasmin Ferdous, Zeenat Jabin, Papia Akhter and Fatima Begum \\ National Institute of Nuclear Medicine \& Allied Sciences (NINMAS)
}

Correspondence Address: Dr. Jasmin Ferdous. Assistant Professor \& SMO, Thyroid Division, NINMAS, BSMMU Campus, Shahbag, Dhaka-1000. jasmin.ferdous.aelee@gmail.com.

\begin{abstract}
Follicular carcinoma of thyroid usually behaves in an indolent manner with low metastatic potential. Distant metastases as initial presentation are rare in follicular carcinoma, especially in young patients. Blood borne metastasis is common with spread to lung, bone and other solid organs. However, metastatic mediastinal tumors are rare.

Here, a neglected case of follicular carcinoma of thyroid (FTC) is reported. The patient is a 43 year old female who was referred to the Thyroid Division of NINMAS for radioiodine therapy for an inoperable metastatic FTC. She was presented with fever, shortness of breath and chest discomfort. $99 \mathrm{~m} \mathrm{Tc}$ scan showed concentration of most of the isotope in the big metastatic mediastinal mass. Because of the sheer volume of the mass, a plan was undertaken to first reduce her tumor burden with external beam radiation therapy (EBRT) and then considered adjuvant therapy with radioiodine after manageable regression in size of the metastatic tumor. She had a history of thyroidectomy for FTC 14years back without radio iodine ablation.

Negligence about proper management for FTC may result in poor outcome like huge mediastinal mass as in this reported case. Radioiodine therapy is usually the first line approach for functional metastasis, but in exceptional cases when the tumor size is very big, EBRT may be considered first. Radiotherapy (RT) is effective in relieving compression symptoms and may improve the quality of life in these patients..
\end{abstract}

Key words: Follicular Thyroid Carcinoma, Mediastinal mass, Metastasis

Bangladesh J. Nucl. Med. Vol. 22 No. 2 July 2019

Doi: https://doi.org/10.3329/bjnm.v22i2.51767

\section{INTRODUCTION}

Follicular Thyroid Carcinoma (FTC) is the second most prevalent of the thyroid carcinomas and it usually spreads by hematogenous route. The treatment protocol for FTC consists of surgery followed by ablation with radioactive iodine. Rarely FTC metastasis occurs in mediastinum and $3 \%$ have been reported as tumors within the chest. However, the incidence of malignancy in mediastinal masses reported ranged from 25 to $49 \%$ (1). Here, an interesting case of FTC is reported with unusual and extensive anterior mediastinal invasion.

\section{CASE REPORT}

A 43 year old female noticed a swelling in the neck and visited a physician 14 years back. She had no past medical history or risk factors for thyroid malignancy. Laboratory findings and thyroid function tests were normal. Thyroid ultrasonography revealed a predominantly solid, hyopechoic thyroid nodule in left lobe with sonographic features suggestive of malignancy. The right lobe was considered normal. Ultrasound guided FNAC demonstrated nodular goiter without any conclusive information about malignancy. Histopathology report revealed FTC after total thyroidectomy (2002) and the patient was advised to visit Nuclear Medicine (NM) department for RAIT. Patient totally neglected further treatment with RAIT and/or hormone replacement.

Gradually she developed chest discomfort and breathlessness after 12 years (2014) and visited a chest physician. Chest $\mathrm{X}$ ray revealed anterior mediastinal mass with possibility of lymphadenopathy. Contrast CT scan of chest showed multiple enhancing soft tissue density nodules in apico-posterior segment of left upper lobe and also a strongly enhancing soft tissue density mass (about $11 \mathrm{~cm} \mathrm{X} 09 \mathrm{~cm}$ ) with internal calcification in anterior mediastinum. The mass compressed great vessels and pushed the trachea posteriorly. CT guided FNAC revealed metastatic FTC.There was no lung metastasis. Invasion of tumor to ascending aorta, superior vena cava and right atrium could not be excluded but showed compressions. Patient was referred to NINMAS for RAIT as surgeons found her inoperable. Laboratory findings revealed hyperthyroidism (TSH $<0.01 \mathrm{mIU} / \mathrm{L}$ ) and her serum thyroglobulin (Tg) level was found to be more than $300 \mathrm{ng} / \mathrm{ml}$. Thyroid scan (with $99 \mathrm{mTc}$ ) showed no radiotracer concentration in the thyroid bed but almost all radiotracer concentrated in the big mediastinal mass. High resolution ultrasound of neck (HRUS) detected a big (definite measurement was not possible), 
predominantly solid, heterogeneous mass behind the sternum. Since the tumor size was huge, a customized treatment plan was designed to reduce her tumor burden with external beam radiation therapy (EBRT) rather than prepare her for RAIT as a first line approach.
EBRT was chosen as the first line of treatment with two major expectations: 1) it would help to alleviate the compression symptoms that the patient was experiencing 2) it would be effective in regressing the tumor to a manageable size for subsequent radioiodine therapy.
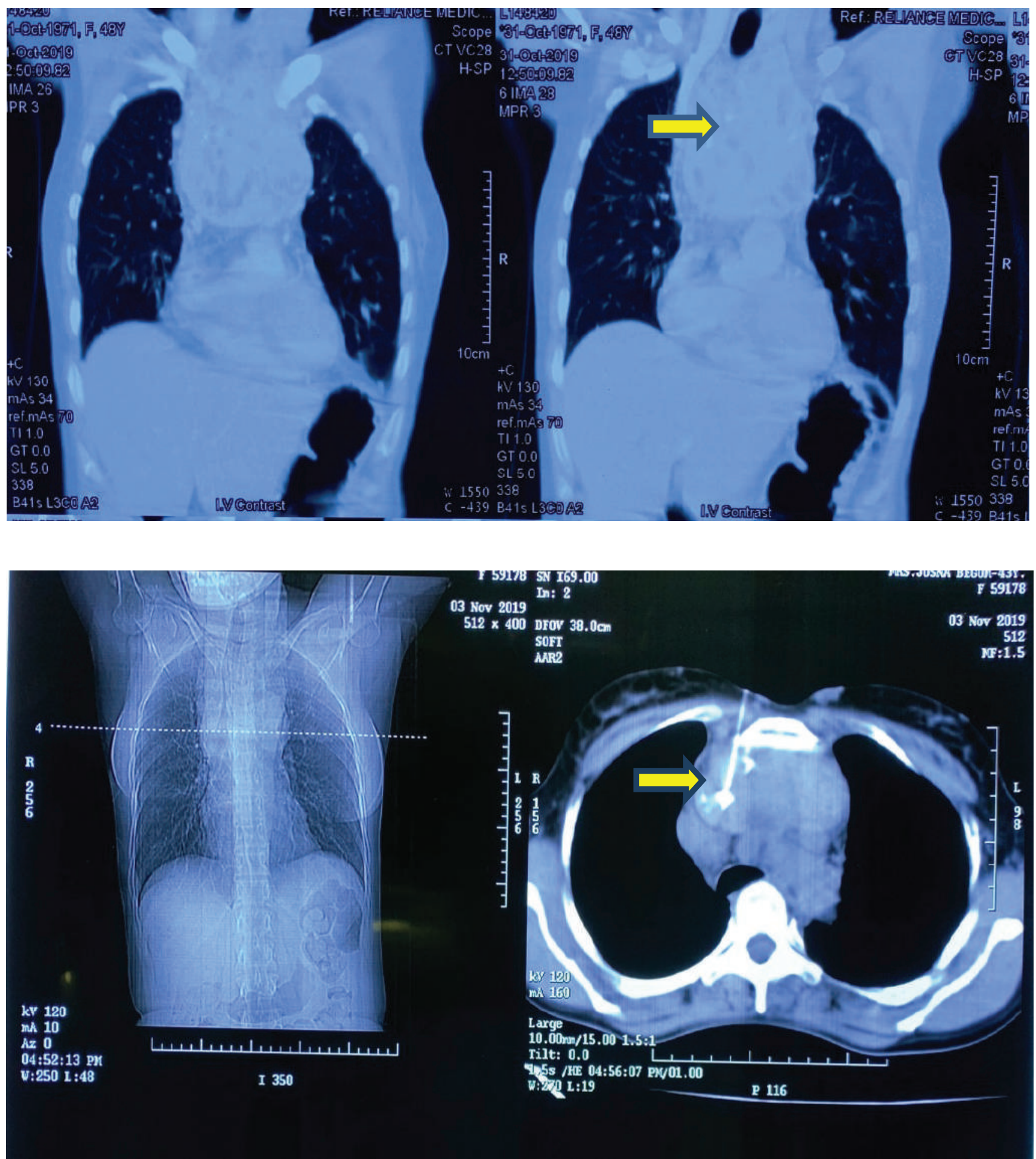

Figure 1: CT image showing enhancing soft tissue density mass $(11 \mathrm{X09} \mathrm{cm})$ located in anterior mediastinum having internal calcifications. 


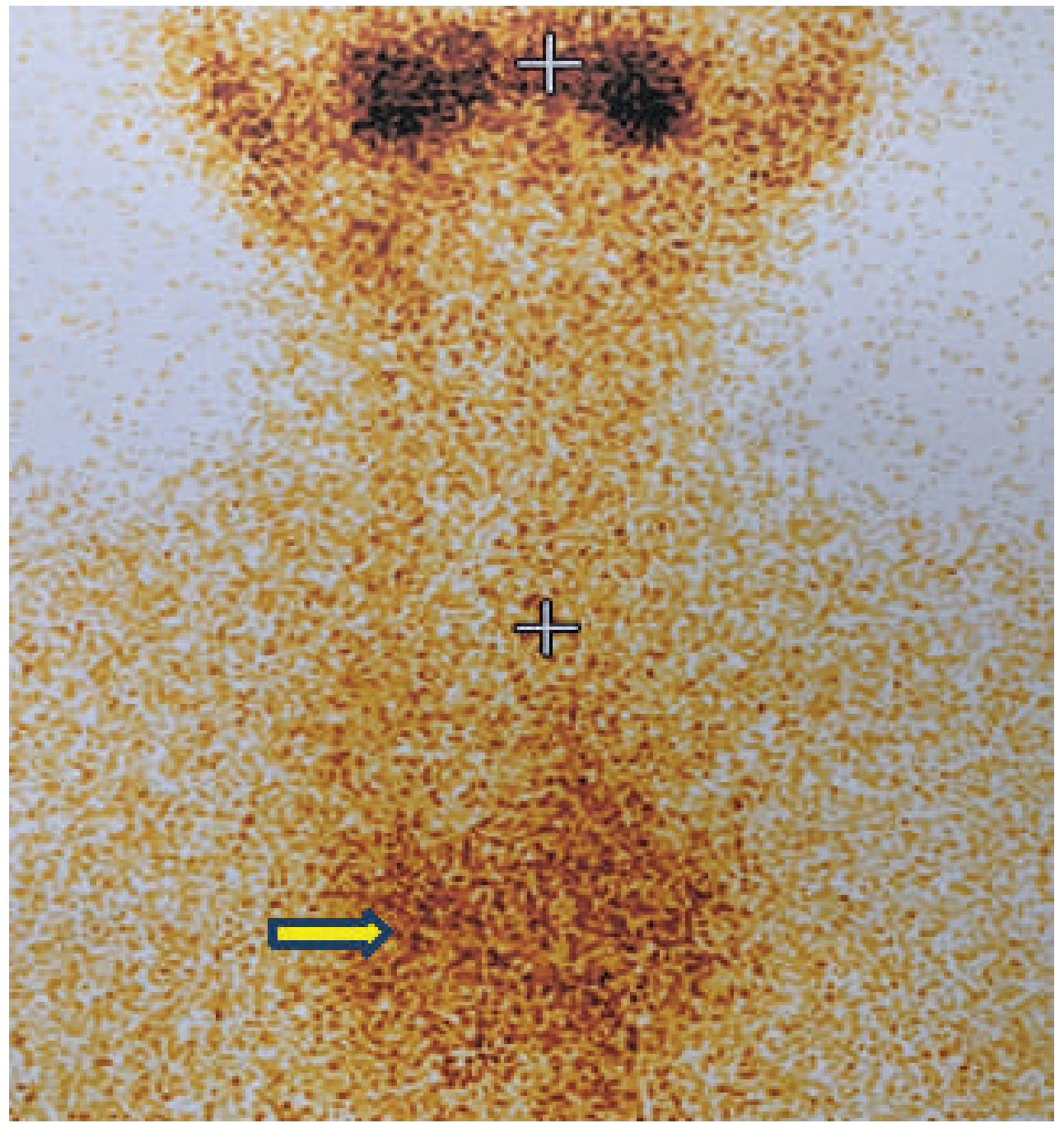

Figure 2: 99mTc scan image showing no radiotracer concentration in thyroid bed. However, a fairly big, irregular shaped area shows slightly non-uniform radiotracer concentration in the anterior mediastinum below thyroid bed.

\section{DISCUSSION}

Follicular carcinoma is the second most prevalent of the thyroid carcinomas with an incidence of approximately $10-15 \%$. Hematogenous metastasis is common with spread to lung, bone and other solid organs. In less than $10 \%$ cases of follicular carcinoma, there is evidence of lymphatic involvement (2). Patients with differentiated thyroid carcinoma (DTC) have a 10-year survival rate of 80-95\%. However, when distant metastases are present, the overall 10 -years survival rate is $40 \%$ (3).

FTC usually behaves in an indolent manner with low metastatic potential. Distant metastases as initial presentation is rare in follicular carcinoma, especially in young patients. The case reported here is unusual since mediastinal tumors are uncommon and represent only $3 \%$ of the tumors seen within the chest (1).

In reports on mediastinal masses, the incidence of malignant lesions ranged from 25 to $49 \%(4,5)$. The anterior mediastinal tumors include teratomas, lymphomas, thymic cancers, thymic carcinoids, thymic cysts and other metastatic tumors. Thyroid cancer rarely metastasizes to the mediastinum and there have been few reports of metastatic mediastinal tumors of thyroid origin. Follicular carcinoma is divided into minimally invasive and invasive variants based on morphologic criteria. Minimally invasive follicular carcinoma is an encapsulated tumor with microscopic penetration of the tumor capsule without vascular invasion $(6,7)$. Patients 
with minimally invasive follicular carcinoma tend to be younger than patients with invasive follicular carcinoma, and it has been suggested that minimally invasive follicular carcinoma may be a precursor to its invasive counterpart (8). Shaha reported an overall survival at 5, 10 , and 20 years for patients with follicular thyroid cancer of $85 \%, 80 \%$ and $76 \%$, respectively (9). When patients with follicular carcinoma were divided into low, intermediate, and high-risk groups based on age, T stage, distant metastases, histologic type, and grade, their survival rates were $98 \%, 88 \%$, and $56 \%$ at 10 years and $97 \%, 87 \%$, and $49 \%$ at 20 years, respectively. D'Avanzo and colleagues reported that patients with minimally invasive follicular carcinoma have a 98\% 10-year survival, compared with $80 \%$ at 10 years for patients with angioinvasive follicular carcinoma with or without capsular invasion and $38 \%$ with extensive invasion of the tumor capsule and the thyroid parenchyma (10). The cause of death is most commonly from progression of distant metastases (11).

The case reported here is remarkable in that she survived more than 14 years without RAIT and/or hormone replacement. But it is evident that the cancer was progressing slowly and insidiously albeit sub-clinically until it became clinical with compression symptoms 12 year later. This slow progression is consistent with the indolent character of the follicular variety of the thyroid cancer.

The compression symptoms that the patient experienced were attributed to the slowly enlarging mediastinal metastasis. This was revealed on CT scan as a strongly enhancing soft tissue density mass having internal calcifications in anterior mediastinum. The mass compressed great vessels and trachea posteriorly. Core biopsy revealed metastatic follicular carcinoma in this patient.

The standard procedure for initial treatment of DTC is surgery followed by radioiodine therapy and hormone replacement. The management plan may differ from patient to patient and depends on several risk factors. However surgery followed by post-operative RAIT has been associated with improved prognosis (12). It is a well established fact that optimal management of patients with complex thyroid cancer requires an integrated team approach involving endocrinologists, NM physicians, medical oncologists, radiation oncologists, and surgeons
(13). Radical surgery, remnant thyroid ablation and RAIT all improve survival, but this is less apparent for patients presenting with distant metastases as this subgroup shows worse prognosis. Accordingly surgery would have been the first choice in the management of the case presented here. However, due to the sheer volume of the mass and tracheal invasion surgical resection was not possible.

EBRT is recommended for palliative purposes to obtain local control for extensive diseases as first line therapy (14). The case reported here was referred to oncologist for EBRT with two goals. (1) To alleviate the compression symptoms (2) regression to a manageable size for subsequent RAIT.

Consequently the patient received a course of conventional radiation with 50 Gy in 25 fractions over 4 weeks targeting on the area volume encompassing the thyroid bed and the gross disease. She tolerated EBRT very well and her stridor subsided. There was tracheal inflammation and skin erythema which are expected side-effects of the radiotherapy, but these were transient. She also had complaints of dysphagia, but did not require a feeding tube. The patient is now recuperating and radioiodine will be considered as an adjuvant in the treatment plan. However, if RAIT fails patient may be considered for Tyrosine Kinase Inhibitors (TKIs) which is reported to be effective for RAI-refractory DTC (15).

\section{CONCLUSION}

Outcome of DTC is excellent with early diagnosis, meticulous surgery, proper staging, RAIT, lifelong thyroxine supplementation and regular follow- up but it may turn aggressive without appropriate management.

Individualized and an alternative therapeutic approach should be considered in patients of DTC with inoperable, huge metastatic mediastinal mass. Palliative use of EBRT for local control of extensive disease may be considered as first line of therapy; when other modality of treatment cannot be applied. Radiotherapy (RT) seems effective in relieving compression symptoms and may prolong survival and improve QOL.

\section{REFERENCES}

1. Aroor AR, Prakasha SR, Seshadri ST \&Raghuraj U. A study of clinical characteristics of mediastinal mass. Journal of Clinicaland Diagnostic Research 20148 77-80. (https://doi.org/10.7860/JCDR/2014/7622.4013). 
2. Santacroce L, Gagliardi S: Thyroid, follicular carcinoma. Emedicine 25Sep, 2009. Available at

http://emedicine.medscape.com/article/278488-overview (accessed March 30, 2010).Schlumberger MJ. Papillary and follicular thyroid carcinoma. New England J Med1998; 338:297-306.

3. Wongsangiem M, Tangthangtham A, Primary tumours of the mediastinum: 190 cases analysis (1975-1995) J Med Assoc Thai 1996 79:689-97. [Google Scholar]

4. Temes R, Chavez T, Mapel D, Ketai L, Crowell R, Key C, Primary mediastinal malignancies: findings in 219 patients West J Med 1999 170:161-6.

5. Azarow KS, Pearl RH, Zurcher R, Edwards FH, Cohen AJ, Primary mediastinal masses. A comparison of adult and pediatric populations $\mathrm{J}$ Thorac Cardiovasc Surg 1993 106:67-72.

6. D'Avanzo A, Treseler P, Ituarte PH, et al. Follicular thyroid carcinoma: Histology and prognosis. Cancer. 2004; 100:1123-1129.

7. Collini P, Sampietro G, Rosai J, et al. Minimally invasive (encapsulated) follicular carcinoma of the thyroid gland is the low-risk counterpart of widely invasive follicular carcinoma but not of insular carcinoma. Virchows Arch. 2003; 442:71-76.

8. Serra S, Asa SL. Controversies in thyroid pathology: the diagnosis of follicular neoplasms. EndocrPathol. 2008; 19:156-165.
9. Shaha AR. Implications of prognostic factors and risk groups in the management of differentiated thyroid cancer. Laryngoscope. 2004; 114:393-402.

10. D'Avanzo A, Treseler P, Ituarte PH, et al. Follicular thyroid carcinoma: Histology and prognosis. Cancer. 2004; 100:1123-1129. [PubMed] [Google Scholar]

11. Kitamura Y, Shimizu K, Nagahama M, et al. Immediate causes of death in thyroid carcinoma: clinicopathological analysis of 161 fatal cases. J ClinEndocrinolMetab. 1999; 84:4043-4049. [PubMed] [Google Scholar]

12. Schmidbauer B, Menhart K, Hellwig D, Grosse J. Differentiated thyroid cancer - treatment: state of the art. International journal of molecular sciences. 2017 Jun;18(6):1292. https://doi.org/10.3390/ijms18061292]

13. Tuttle M, Robbins R, Larson SM, Strauss HW. Challenging cases in thyroidcancer: a multidisciplinary approach. Eur J Nucl Med Mol Imaging2004;31: 605 - 612

14. A. Nar Demirer, S. Ayturk, N. B. Tutuncu1, A. Gursoy, Y. Pak2, N. G. Demirag,Unresectable Huge Sternal and Mediastinal Metastasisof Follicular Thyroid Carcinoma; Radiotherapy as First-line and Palliative Therapy. Exp Clin Endocrinol Diabetes 2009; 117: 155-158.

15. Iasaki H, Yamazaki H, Takasaki H, Suganuma N, Sakai R, Nakayama H, Hatori S, Toda S, Masudo K. Treatment outcomes of differentiated thyroid cancer with distant metastasis improve by tyrosine kinase inhibitors. Oncology letters. 2019 Jun 1;17(6):5292-300. 